\title{
Mesothelioma of the tunica vaginalis: a series of eight cases with uncertain malignant potential
}

\author{
Fadi Brimo $^{1}$, Peter B Illei ${ }^{1}$ and Jonathan I Epstein ${ }^{1,2,3}$ \\ ${ }^{1}$ Department of Pathology, The Johns Hopkins Hospital, Baltimore, MD, USA; ${ }^{2}$ Department of Urology, \\ The Johns Hopkins Hospital, Baltimore, MD, USA and ${ }^{3}$ Department of Oncology, The Johns Hopkins \\ Hospital, Baltimore, MD, USA
}

\begin{abstract}
Well-differentiated papillary mesotheliomas have rarely been reported to involve the testis tunica vaginalis. While the classic histology of the originally described well-differentiated papillary mesotheliomas consisted of papillae lined by a single layer of bland cuboidal cells, more complex architectural patterns have been described. This report details our experience with eight paratesticular mesotheliomas that span the histological spectrum from classic well-differentiated papillary mesotheliomas to those with more complex patterns. We attempt to determine whether there are prognostic or immunohistochemical differences between these lesions and 28 diffuse malignant mesotheliomas. All cases had papillary/tubulopapillary in addition to more complex architectures (cribriform, condensed) and none showed evidence of invasion. Mitotic figures were present in seven cases and averaged 2.1 mitosis per 50 h.p.f. All cases showed $\leq 1 \%$ of positive cells staining for Ki-67 and p53, and only one case stained positive for GLUT-1 in comparison with $50 \%$ positivity for GLUT-1 in diffuse mesotheliomas. Of five patients with follow-up information of more than 1 year, three patients were alive at 2, 3 and 9 years, and two died of unknown causes at 5 and 47 years, suggesting that these lesions behave more indolently than typical malignant mesotheliomas of the tunica vaginalis. If these cases were diffuse malignant mesotheliomas, one would have expected at least some of the patients to have died of disease within a couple of years after diagnosis. However, detailed and long-term follow-up were not sufficiently available to reach definitive conclusions on the true biological behavior of these tumors. The morphological continuum noted between our cases and classic well-differentiated papillary mesotheliomas, combined with their immunohistochemical profile and indolent behavior, contrasts with that of diffuse malignant mesotheliomas. We propose that these tunica vaginalis mesotheliomas, which are more complex than the classic well-differentiated papillary mesotheliomas, and yet are not overtly histologically malignant, be classified as 'mesotheliomas of uncertain malignant potential'.

Modern Pathology (2010) 23, 1165-1172; doi:10.1038/modpathol.2010.113; published online 4 June 2010
\end{abstract}

Keywords: GLUT-1; mesothelioma; paratesticular; tunica vaginalis

Mesotheliomas are relatively rare tumors that arise from the serosal surface of the pleura, peritoneum and pericardium. On rare occasions, they originate from the tunica vaginalis of the testis in which case they manifest as a paratesticular mass. Although diffuse malignant mesotheliomas are

Correspondence: Dr JI Epstein, MD, Department of Pathology, Johns Hopkins Medical Institution, 401 N Broadway, Weinberg Building Room 2242, Baltimore, MD 21231, USA.

E-mail: jepstein@jhmi.edu

Received 14 February 2010; revised 31 March 2010; accepted 1 April 2010; published online 4 June 2010 highly malignant tumors, rare forms that are much less aggressive, exist. These include multi-cystic mesotheliomas and well-differentiated papillary mesotheliomas. ${ }^{1,2}$ Well-differentiated papillary mesotheliomas usually occur in the peritoneum of young women, but on rare occasions they have been reported to involve the testis tunica vaginalis. While the histology of the originally described classic welldifferentiated papillary mesothelioma consisted of papillae lined by a single layer of bland cuboidal cells, more complex architectural patterns of growth, including tubulopapillary and even solid patterns, have been described and included by some 
investigators as part of the spectrum seen with this entity. ${ }^{1-3}$ This report details our experience with eight paratesticular mesotheliomas that have histological features that are borderline between classic well-differentiated papillary mesothelioma and diffuse malignant mesothelioma. We also attempt to determine whether there are immunohistochemical differences between these borderline cases and overt diffuse malignant mesothelioma.

\section{Materials and methods}

Eight cases of paratesticular mesotheliomas with borderline histological features between welldifferentiated papillary mesothelioma and diffuse malignant mesothelioma were identified from the consultation files of one of the authors. Although there has been a controversy in the literature regarding the definition of well-differentiated papillary mesothelioma, for the purposes of this study and as defined by most investigators in the literature, we use this term for a localized solitary tumor exhibiting exclusively a papillary architecture in which the papillae are lined by a single layer of bland cuboidal cells. Clinical information, including treatment and follow-up, was obtained when available by contacting the treating physician. In all cases, death status was searched 'online' using the Social Security Death Index Database website (http://www.genealogybank.com/gbnk/ssdi/). Hematoxylin and eosin-stained slides were available for review in each case. Immunohistochemical staining was performed on formalin-fixed, paraffin-embedded tissue in all cases using an avidin-biotin peroxidase complex method. Sections from the paraffin block were cut at $4-5 \mu \mathrm{m}$, placed on positively charged slides, deparaffinized in organic solvents, treated with methamolic $\mathrm{H}_{2} \mathrm{O}_{2}$ and rehydrated. P53 (1:2000, monoclonal; Ventana), Ki-67 (1:1000, monoclonal; Ventana) and GLUT-1 (1:100, monoclonal; Dako) stains were performed. Immunoreactivity was visualized using diaminobenzidine as the chromogen. We also included in this study 28 cases of diffuse malignant mesotheliomas taken from a recently constructed tissue microarray that included pleural-based mesotheliomas. Those cases were stained with p53 and GLUT-1 to compare their staining pattern with cases from this series. Ki-67 staining on the pleural mesotheliomas' tissue microarray was attempted but was unsuccessful because of the lack of tissue. The percentage of cells showing nuclear staining for p53 and Ki-67, and membranous staining for GLUT-1, was recorded by visual estimation, and a case was considered positive for p53 only when strong nuclear staining was noted.

\section{Results}

\section{Clinical Features}

The salient clinical features of cases are summarized in Table 1. The age of patients at presentation ranged from 34 to 73 years (mean 52.5). In seven out of eight cases, hydrocele was the initial manifestation of disease, and only one patient (no. 4) presented with a scrotal mass. Detailed history of asbestos exposure was unavailable.

\section{Pathological Findings}

In six cases (no. 1, 3, 4, 6, 7, 8) the tumor presented as a solitary polypoid papillary mass protruding out of the surface of the tunica vaginalis, and in one case multiple separate nodules were present (no. 5) (Figures 1a and b). In one case (no. 2), the tumor formed a plaque-like lesion (Figure 1c). The greatest size of the tumor (or of the largest lesion in multifocal cases) ranged from 0.3 to $2 \mathrm{~cm}$ (mean $1 \mathrm{~cm}$ ).

All cases had a papillary/tubulopapillary architecture at least focally (occupying 10-95\% of the tumor) (Figure 1d). The fibrovascular papillary cores were of different sizes, with the stroma being hypocellular edematous, myxoid or with hypocellular fibrosis. In all cases, at least focally the papillae were lined by a single layer of cuboidal cells (Figure 1e). In six cases, the papillary projections

Table 1 Clinical features

\begin{tabular}{|c|c|c|c|c|}
\hline Case no. & Age (years) & Clinical presentation & Treatment & Follow-up \\
\hline 1 & 43 & Hydrocele & Hydrocelectomy & $\begin{array}{l}3 \text { years, NED } \\
9 \text { years, A }\end{array}$ \\
\hline 2 & 49 & Hydrocele & Orchiectomy & $\begin{array}{l}1.5 \text { years, NED } \\
2 \text { years, } \mathrm{A}\end{array}$ \\
\hline 3 & 73 & Hydrocele & Orchiectomy & $\begin{array}{l}2 \text { months, NED } \\
8 \text { months, A }\end{array}$ \\
\hline 4 & 34 & Scrotal mass & Orchiectomy & 3 years, $\mathrm{A}$ \\
\hline 5 & 61 & Hydrocele & Hydrocelectomy & $\begin{array}{l}1.5 \text { years, NED } \\
5 \text { years, D }\end{array}$ \\
\hline 6 & 53 & Hydrocele & Hydrocelectomy & 47 years, D \\
\hline 7 & 57 & Hydrocele & Hydrocelectomy & Recent \\
\hline 8 & 50 & Hydrocele & Hydrocelectomy & Recent \\
\hline
\end{tabular}

Abbreviations: A, alive; D, dead unknown causes; NED, no evidence of disease. 
a

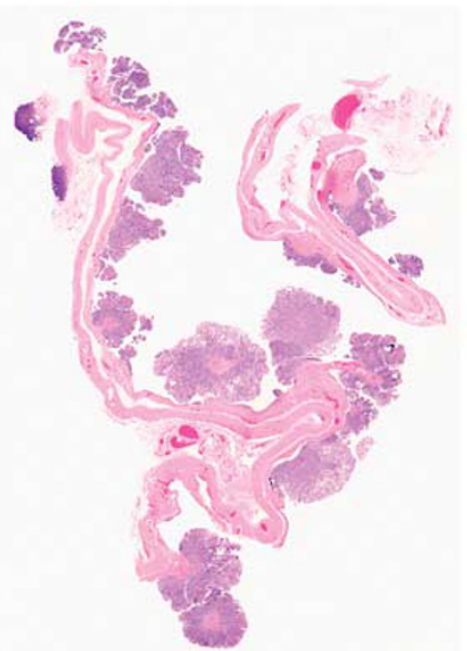

d

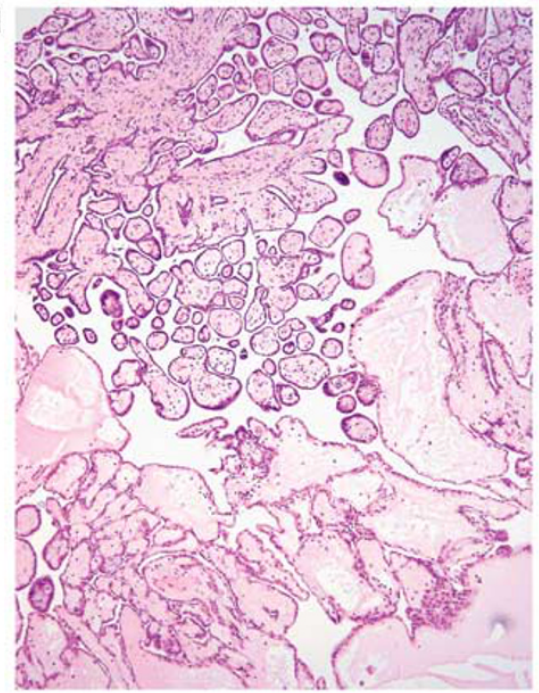

b

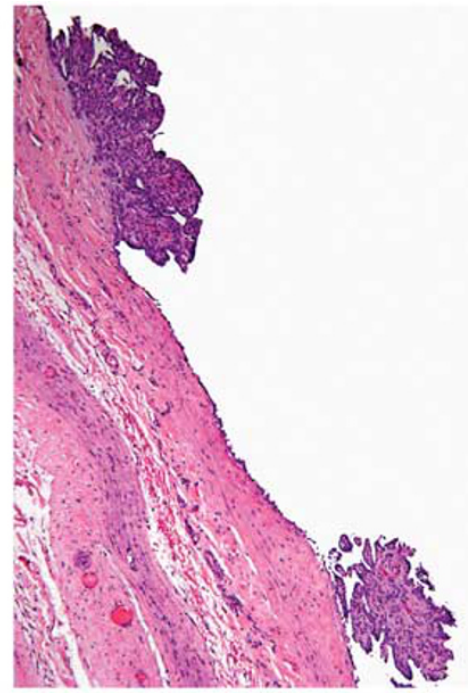

e

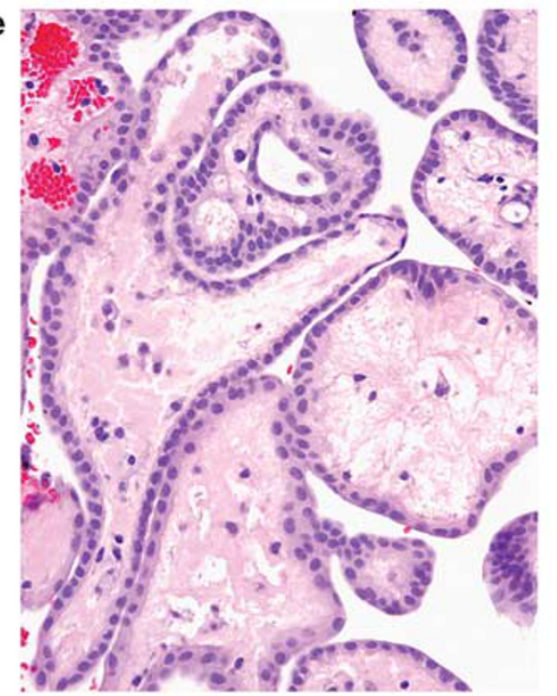

C



f

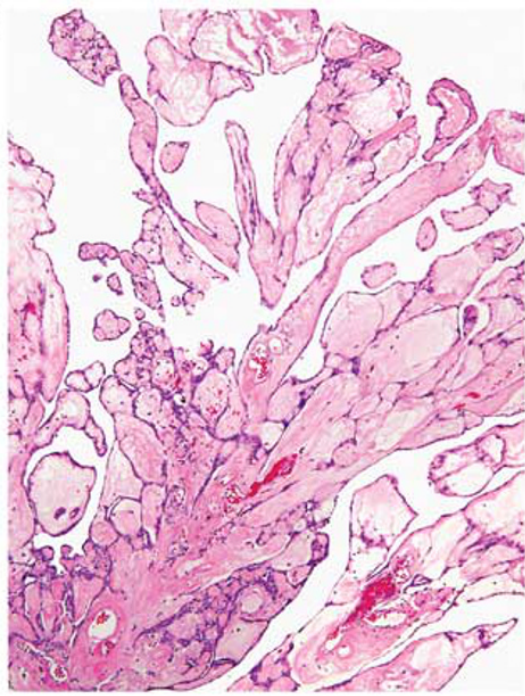

Figure 1 (a) A low-magnification view of multiple papillary masses studding the surface of the tunica vaginalis. (b) Two small papillary excrescences of mesothelioma on tunica vaginalis. (c) Plaque-like growth of mesothelioma seen in one case. (d) A low-magnification view of the papillary growth pattern of mesothelioma. (e) A high-magnification view of mesothelioma showing single layer of bland cuboidal cells. (f) In addition to papillary projections, the base of the stalks shows hyalinization with anastomosing cords of tumor cells forming cribriform structures.

also contained anastomosing cords of tumor cells that formed cribriform structures (Figures 1f-2a). In some areas, the cords had a back-to-back arrangement with minimal intervening stroma, showing a condensed appearance (seven cases), and in two cases (no. 2 and 5) this was the predominant pattern in the tumor (Figures 2a and b). One case had prominent acellular sclerosis (Figure 2c) and in another case (no. 6) psammoma bodies were identified. In one case (no. 8) the tumor contained a wellcircumscribed area that was indistinguishable from giant cell tumor of bone/soft tissue, with a proliferation of monotonous mononuclear cells and osteoclast-like giant cells in a background of hemorrhage and hemosiderin-laden macrophages. While the osteoclast-like giant cells stained positive for CD68, the mononuclear cells stained focally positive with epithelial and mesothelial markers (Figure 2e and f). In all the cases, despite the complex architectural patterns, none showed clear-cut evidence of invasion of the sub-mesothelial tissue, lacking downward irregular infiltrative borders or a surrounding cellular desmoplastic stromal reaction.

Cytologically, individual tumor cells in six cases showed scattered mild degenerative atypia, with moderately abundant eosinophilic cytoplasm, central vesicular nuclei and occasional conspicuous nucleoli (Figure 2d). In the other two cases, the nuclei were uniformly bland. Nuclear pseudoinclusions were abundant and were identified to some extent in all cases. Mitotic figures were present in seven out of eight cases, and averaged 2.1 mitosis per 50 high-power field (h.p.f.) (range 0-6) (Figure 2d). In the case that showed the highest mitotic activity 
a

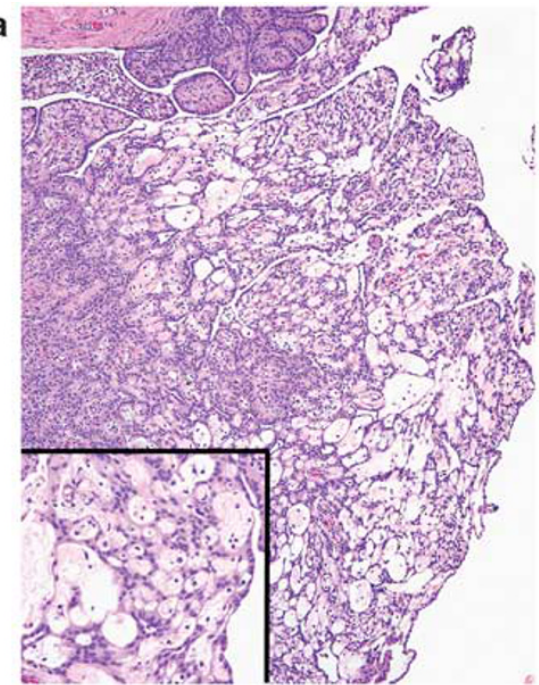

d

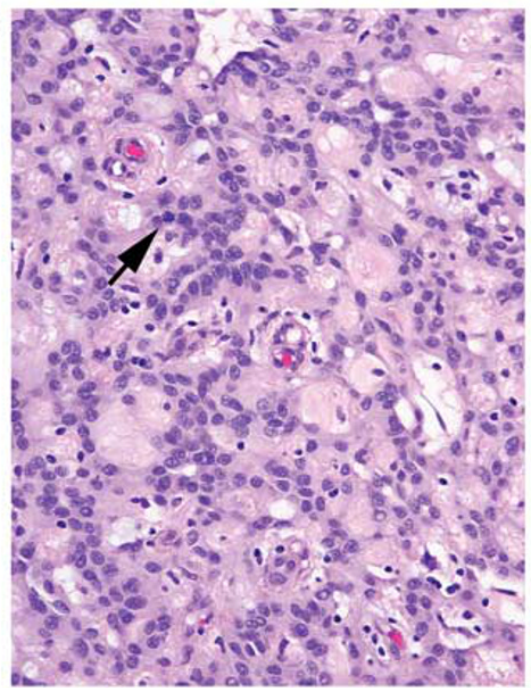

b

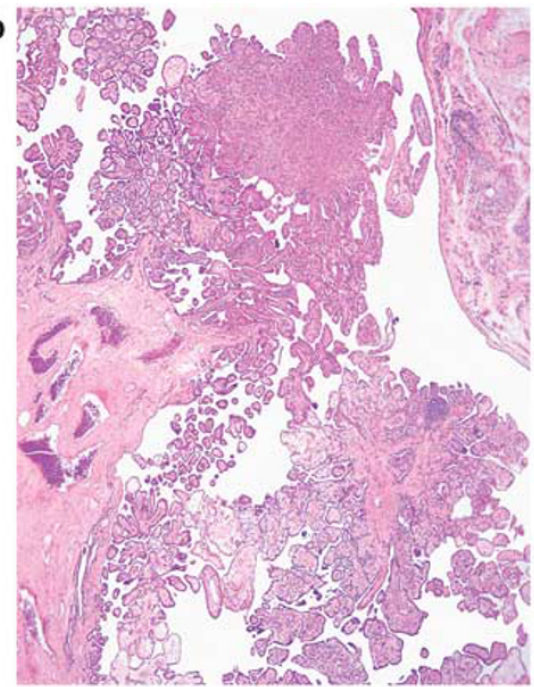

e



c
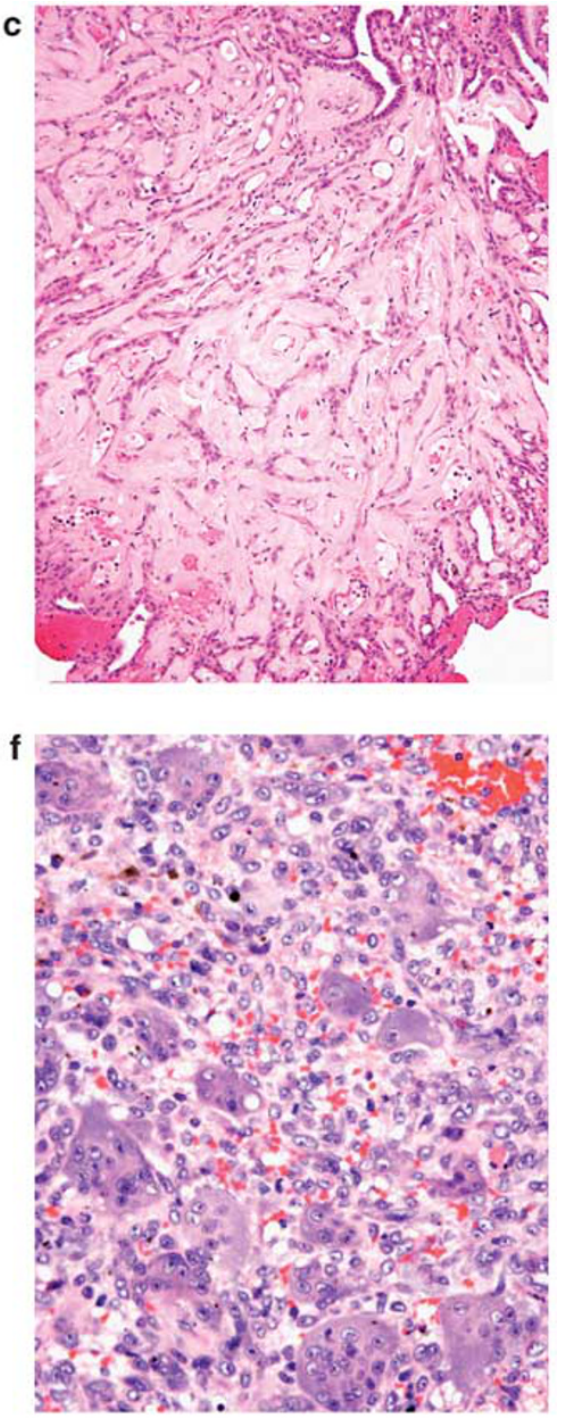

Figure 2 (a) Anastomosing cords of tumor cells forming cribriform (top and inset) and condensed growth patterns (bottom) alternating with the papillary architecture (left). (b) The spectrum of patterns ranging from papillary (left) to cribriform and condensed. (c) In one case, cells were surrounded by a prominent acellular sclerotic stroma, resulting in a pseudo-infiltrative growth pattern. (d) The individual tumor cells may show mild degenerative atypia with occasional conspicuous nucleoli and mitotic figures (arrow). (e) Paratesticular mesothelioma (right) admixed with a well-circumscribed area rich in giant cells. (f) A giant cell tumor-like area composed of monotonous mononuclear cells and osteoclastic giant cells.

(no. 3), mitoses were mainly seen in the areas of the tumor with complex architecture (cribriform, or condensed patterns of growth) rather than in the cells lining the papillary structures. All cases lacked atypical mitotic figures or necrosis (Table 2).

\section{Immunohistochemical Findings}

Only one of the eight cases of the series $(12.5 \%$ of cases) stained positive for GLUT-1 and the staining was very focal ( $1 \%$ of the cells). In comparison, $50 \%$ $(n=14)$ of the cases of diffuse malignant mesotheliomas were positive for GLUT-1, with the majority showing less than $25 \%$ positivity for the cells. The same pattern was noted for Ki-67, which labeled $\leq 1 \%$ of the cells in all cases of our tunica vaginalis mesotheliomas. p53 was negative in five cases of tunica vaginalis mesotheliomas and $\leq 1 \%$ in the remaining three cases in comparison with $31 \%$ positivity ( 8 out of 26 evaluable cases) in the diffuse malignant mesothelioma cases with a mean of $33 \%$ positive cells in individual cases (range 1-90\%).

\section{Treatment and Follow-up}

Three patients underwent radical orchiectomy, which showed no evidence of residual disease. In five patients the only surgical procedure was a 
Table 2 Pathological findings

\begin{tabular}{|c|c|c|c|c|c|}
\hline Case no. & Focality & Size $(\mathrm{cm})$ & $\begin{array}{c}\text { Architectural } \\
\text { pattern of growth }\end{array}$ & Atypia & $\begin{array}{l}\text { Mitotic count } \\
\text { (per } 50 \text { h.p.f.) }\end{array}$ \\
\hline 1 & Solitary & 0.3 & $\begin{array}{c}50 \% \text { Pap } \\
50 \% \text { C } \\
20 \% \text { Cr }\end{array}$ & Mild & 2 \\
\hline 2 & Multi-focal & 1.1 & $\begin{array}{c}90 \% \text { C } \\
10 \% \text { Pap }\end{array}$ & Mild & 2 \\
\hline 3 & Solitary & 2 & $\begin{array}{c}75 \% \text { Pap } \\
20 \% \text { Cr } \\
5 \% \text { C }\end{array}$ & No & 6 \\
\hline 4 & Solitary & 1.4 & $\begin{array}{l}95 \% \text { Pap } \\
5 \% \text { C }\end{array}$ & Mild & 1 \\
\hline 5 & Multi-focal & 0.3 & $\begin{array}{c}50 \% \mathrm{C} \\
30 \% \mathrm{Cr} \\
20 \% \text { Pap }\end{array}$ & Mild & 1 \\
\hline 6 & Solitary & 0.7 & $\begin{array}{c}90 \% \mathrm{Cr} \\
10 \% \text { Pap }\end{array}$ & No & 0 \\
\hline 7 & Solitary & 0.6 & $\begin{array}{c}30 \% \text { Pap } \\
60 \% \text { Cr } \\
10 \% \text { C }\end{array}$ & Mild & 3 \\
\hline 8 & Solitary & 1.3 & $\begin{array}{c}80 \% \text { Cr } \\
10 \% \text { C } \\
10 \% \text { Pap }\end{array}$ & Mild & 2 \\
\hline
\end{tabular}

Abbreviations: C, condensed; Cr, cribriform; Pap, papillary; h.p.f, high-power field.

hydrocelectomy. None of the patients received adjuvant chemotherapy or radiation therapy. Follow-up information ( $\geq 1$ year) from urologists was available in three of the eight cases. Among the three patients with follow-up information from urologists, all did not show evidence of disease recurrence or progression at 1.5 years (two patients) and 3.5 years (one patient) post-surgery; on the basis of the Social Security Death Index, two were alive at 2 and 9 years, and the third died of unknown causes after 5 years. In addition, on the basis of the Social Security Death Index, one of the eight patients was alive (disease status unknown) at 3 years and another patient died of unknown causes 47 years following diagnosis. The three remaining cases were recent; in one case, the patient was free of disease 2 months after surgery, and two cases are too recent for follow-up.

\section{Discussion}

During embryonic life, the tunica vaginalis forms from an outpouching of the peritoneal fold and thus mesotheliomas may rarely develop in the paratesticular tissues. ${ }^{4-6}$ Paratesticular mesotheliomas, which comprise less than $1 \%$ of all cases of mesothelioma, are usually malignant and aggressive tumors that are morphologically identical to mesotheliomas at other sites, and may therefore show an epithelial, spindle cell or biphasic morphology. ${ }^{1,2,7}$ Well-differentiated papillary mesothelioma is a rare form of mesothelioma that typically arises from the peritoneum or pleura and only rarely involves the testicular tunica vaginalis. Differentiat- ing diffuse malignant mesothelioma from welldifferentiated papillary mesothelioma has prognostic and therapeutic implications.

In the literature, different morphological criteria for what constitutes a well-differentiated papillary mesothelioma have been described over the years, which have contributed in major part to the controversy surrounding this entity in terms of the terminology used and the behavior of those tumors. The definition provided by most experts in the vast majority of cases, including those in the World Health Organization (WHO) classification of tumors and in three of the four largest published series, is that of a localized solitary tumor showing exclusively a papillary architecture in which the papillae are lined by a single layer of bland cuboidal cells. ${ }^{2,8-12}$ In other reports, a tubular architecture was included as part of the histological spectrum of changes that can be seen in this entity. ${ }^{1,3,6}$ In the original series of Daya et al, some cases with more complex architectural patterns, including focal solid growth, were also described. However, the authors acknowledged that the lack of long-term follow-up in their series precluded reaching definite conclusions on whether those cases with more complex architecture can be considered with certainty as welldifferentiated papillary mesotheliomas, and therefore as indolent tumors, or whether they should be considered as diffuse mesotheliomas. ${ }^{3}$ The common histological features in all the reported cases of welldifferentiated papillary mesotheliomas are the bland nuclear cytology and low or absent $(\leq 1$ mitotic figure per 10 h.p.f.) mitotic activity. In a series of 19 peritoneal mesotheliomas in women, Goldblum et al reported six cases of incidental, small and localized 
peritoneal mesothelial neoplasms with some morphological features similar to those reported in the current series: one was exclusively composed of papillary structures lined by bland cells and five had a tubulopapillary architecture, with two cases showing small foci of nests and cords of neoplastic cell in the sub-mesothelial tissue. Nuclei were bland, with the exception of two cases in which grade 2 nuclei were present, and four out of their six cases had mitotic activity that ranged from 1 to 3 mitoses per 10 h.p.f. in 'selected areas of the tumors'. Although all of their cases had a benign follow-up, the authors preferred using the term 'localized mesothelioma' or 'localized mesothelioma of low-grade malignancy' rather than 'well-differentiated papillary mesothelioma,' cautioning of the potential 'benign' connotation (and therefore the potential under-treatment) that a diagnosis of 'welldifferentiated papillary mesothelioma' might give to similar cases without a thorough and long-term clinical follow-up. ${ }^{11}$

Therefore, there seems to be a consensus among different authors that cases, which are exclusively composed of papillary structures lined by bland cells, can be labeled as well-differentiated papillary mesotheliomas and can be expected to follow a benign clinical course if completely excised. However, there is disagreement on how to classify cases that show any of the following features: (1) tumor multi-focality, (2) intermediate-grade nuclei, (3) increased mitotic activity and (4) growth patterns more complex than the simple papillary architecture (similar to the cases reported in the current series). ${ }^{3,11,13,14}$ Another point worth mentioning, as Baker et al and Goldblum et al emphasized, is that one should be aware that individual foci within an unequivocal diffuse malignant mesothelioma might be indistinguishable from a well-differentiated papillary mesothelioma. Therefore, it is imperative that most, if not all, of the lesions be removed before such a diagnosis be rendered. ${ }^{11,13}$

To our knowledge, there have only been 11 well-documented cases reported as paratesticular 'well-differentiated papillary mesothelioma' to date. ${ }^{3,6,8,12,15-21}$ However, on the basis of microscopic descriptions and illustrations five had more complex areas within them, similar to the cases reported here. ${ }^{6,12,16,18,20}$ Among the 11 cases, only one recurred and progressed to diffuse malignant mesothelioma. A fact that is frequently overlooked is that the only reported 'malignant' case, which was part of a series of 11 cases of mesotheliomas of the tunica vaginalis by Jones et al, was not originally labeled by the authors as a well-differentiated papillary mesothelioma, but rather as a "mesothelioma with prominent papillary features'. ${ }^{20}$ However, it is often referred to in the literature as an example of paratesticular well-differentiated papillary mesothelioma with an aggressive behavior. ${ }^{2,6,20}$ In the original article, this tumor was described as having 'focal microscopic invasion of the hydrocele wall,' which is a finding not usually seen in welldifferentiated papillary mesotheliomas; this case was not illustrated in the paper. ${ }^{20}$ Therefore, it is unknown whether the only case of paratesticular 'well-differentiated papillary mesothelioma' that was reported to have recurred and progressed represents diffuse malignant mesothelioma with prominent papillary features rather than well-differentiated papillary mesothelioma.

The cases in the current series showed morphological features bridging classic well-differentiated papillary and diffuse malignant mesothelioma. In addition to the papillary/tubulopapillary architecture, all cases at least focally showed more complex growth patterns. These included cribriform and condensed growth patterns. We are aware that some of these features might be interpreted by some as evidence of sub-mesothelial stromal invasion and therefore of overt malignancy. However, the neoplastic cells in these areas did not show irregular downward growth and lacked a surrounding cellular desmoplastic stromal reaction, and we favor that they represent proliferating mesothelial cells rather than infiltration. In addition, the giant cell tumorlike growth that was noted in one case from the current series (no. 8) is a feature that has never been reported earlier. Although giant cell tumor of bone/soft tissue follows an indolent course with the potential for local recurrence and very rarely malignant behavior, it is unknown whether the presence of such areas in the current example carries an adverse prognosis. That the giant cell tumor-like areas were intimately admixed with the neoplastic mesothelial cells as part of the same lesion and that some cells within the giant cell tumor-like areas stained focally positive for mesothelial markers make us favor the position that this pattern is a peculiar stromal reaction to mesothelial cells rather than being a separate primary tumor. Other morphological features in the current series that are borderline between welldifferentiated papillary mesothelioma and diffuse malignant mesothelioma are the presence of mitotic activity in the vast majority of cases ( 7 out of 8 cases), in contrast to only one of the 60 cases of welldifferentiated papillary mesothelioma included in the three major series recorded to date. ${ }^{3,10,15}$ It should be noted that mitoses in our cases were relatively rare and averaged 2.1 mitosis per 50 h.p.f., and in no case atypical mitotic figures were seen.

At the immunohistochemical level, no study to date has compared the expression of different markers between diffuse malignant mesothelioma and well-differentiated papillary mesothelioma. Rather, studies have focused on differentiating diffuse malignant mesothelioma from reactive mesothelial proliferations. Although no single antibody has been shown to carry a discriminatory value, several markers have been reported to be more commonly expressed in diffuse malignant mesothelioma than in reactive mesothelium. King et al, who 
analyzed the results of 10 previous studies conducted on the subject, showed that p53 carries a sensitivity of $58 \%$ and specificity of $91 \%$ for diffuse malignant mesotheliomas, and in the majority of those studies, positive p53 staining was considered to be present when any positive staining was seen in an individual case regardless of the percentage of positive cells. ${ }^{22-26}$ Using this latter criterion, three out of our eight cases of tunica vaginalis mesotheliomas showed positivity in comparison with 8 out of 26 evaluable cases (31\% of cases) of pleural diffuse malignant mesothelioma that we studied. However, the percentage of cell staining was significantly different between the two groups; whereas the mean of positive cells was 33\% in diffuse mesothelioma (range 1-90\%), only extremely focal staining ( $\leq 1 \%$ positive cells) was noted in the three positive cases of our series. The same applies to Ki-67, which has shown in the literature higher expression in diffuse malignant mesotheliomas (mean: $24.6 \%$ positive cells; range: $1-70 \%$ ) in comparison with reactive mesothelial cells (mean: $6.2 \%$ positive cells; range: $0-25 \%) .{ }^{27-29}$ GLUT-1, a member of the glucose transporter family, has also recently been reported to be a useful marker in that differential diagnosis by showing higher expression in diffuse malignant mesotheliomas $(63-100 \%$ of cases) in comparison with reactive cases $(0-27 \%$ of cases). ${ }^{30,31}$ In our hands, GLUT-1 was positive in $50 \%$ of the diffuse malignant mesothelioma cases. Only one case of tunica vaginalis mesothelioma was labeled with GLUT-1 and did so only very focally. The immunoprofiles of our cases are closer to that of reactive mesothelial cells than that of diffuse malignant mesotheliomas.

Although a substantial proportion of peritoneal diffuse malignant mesotheliomas have been shown to behave indolently unlike their pleural counterparts, these tumors are still lethal with $60 \%$ of patients dying of the disease in the first 4 years after diagnosis, according to the largest series reported to date by Kerrigan et al. ${ }^{14}$ Furthermore, one cannot necessarily equate the behavior of diffuse peritoneal malignant mesotheliomas in women with tunica vaginalis mesotheliomas in men. Malignant mesothelioma of the tunica vaginalis is reported to carry a mean disease-specific survival ranging from 26 to 36 months in comparison with 6 to 12 months for pleural mesothelioma. ${ }^{32-34}$ Of our five patients with follow-up information over 1 year, patients were alive at 2, 3 and 9 years, and died of unknown causes at 5 and 47 years, suggesting that these lesions behave more indolently than typical malignant mesotheliomas of the tunica vaginalis. If these cases were diffuse malignant mesotheliomas, one would have expected at least some of the patients to have died of disease within a couple of years after diagnosis. However, detailed and long-term followup on these cases was not sufficiently available to reach definitive conclusions on the true biological behavior of these tumors.
We propose restricting the term 'well-differentiated papillary mesothelioma' in the paratesticular region to those localized tumors that show an exclusive papillary architecture in which the papillae are lined by a single layer of bland cuboidal cells. Cases from the current series represent a morphological continuum between classic welldifferentiated papillary mesothelioma and diffuse malignant mesothelioma. Although the evidence suggests that these lesions should not be labeled as diffuse malignant mesothelioma on the basis of immunohistochemical and follow-up data, we do not believe that there are sufficient data in the literature or in the current series to recommend labeling of those tumors as well-differentiated papillary mesothelioma. If additional data become available showing that a minority of these cases is associated with more aggressive behavior, then the term 'mesothelioma of low malignant potential' could be applied. However, on the basis of the current data, we propose that tunica vaginalis mesotheliomas, which are more complex than the classic well-differentiated papillary mesotheliomas, and are yet not overtly histologically malignant, be classified as mesotheliomas of uncertain malignant potential.

\section{Disclosure/conflict of interest}

The authors declare no conflict of interest.

\section{References}

1 Churg A, Cagle PT, Roggli VL. Well differentiated papillary mesothelioma. In: Silverberg SG, Sobin LH (eds). AFIP Atlas of Tumor Pathology: Tumors of the Serosal Membranes, Fourth Series, Fascicle 3 ARP PRESS: Silver Spring, Maryland, 2006, pp 107-109.

2 Davis CJ, Woodward PJ, Dehner LP, Jones MA, Srigley JR, Sesterhenn IA, Gerald WL, Miettinen M, Fetsch JF. Benign mesothelioma. In: Eble JN, Sauter G, Epstein JI, Sesterhenn IA (eds). World Health Organization Classification of Tumours. Pathology and Genetics of the Urinary System and Male Genital Organs. IARCPress: Lyon, 2004, pp 269.

3 Daya D, McCaughey WT. Well-differentiated papillary mesothelioma of the peritoneum. A clinicopathologic study of 22 cases. Cancer 1990;65:292-296.

4 Gupta NP, Agrawal AK, Sood S, Hemal AK, Nair M. Malignant mesothelioma of the tunica vaginalis testis: a report of two cases and review of literature. J Surg Oncol 1999;70:251-254.

5 Plas E, Riedl CR, Pfluger H. Malignant mesothelioma of the tunica vaginalis testis: review of the literature and assessment of prognostic parameters. Cancer 1998;83:2437-2446.

6 Tolhurst SR, Lotan T, Rapp DE, Lyon MB, Orvieto MA, Gerber GS, Sokoloff MH. Well-differentiated papillary mesothelioma occurring in the tunica vaginalis of the testis with contralateral atypical mesothelial hyperplasia. Urol Oncol 2006;24:36-39. 
7 Attanoos RL, Gibbs AR. Primary malignant gonadal mesotheliomas and asbestos. Histopathology 2000;37: 150-159.

8 Butnor KJ, Sporn TA, Hammar SP, Roggli VL. Welldifferentiated papillary mesothelioma. Am J Surg Pathol 2001;25:1304-1309.

9 Cabay RJ, Siddiqui NH, Alam S. Paratesticular papillary mesothelioma: a case with borderline features. Arch Pathol Lab Med 2006;130:90-92.

10 Galateau-Salle F, Vignaud JM, Burke L, Gibbs A, Brambilla E, Attanoos R, Goldberg M, Launoy G. Welldifferentiated papillary mesothelioma of the pleura: a series of 24 cases. Am J Surg Pathol 2004;28:534-540.

11 Goldblum J, Hart WR. Localized and diffuse mesotheliomas of the genital tract and peritoneum in women. A clinicopathologic study of nineteen true mesothelial neoplasms, other than adenomatoid tumors, multicystic mesotheliomas, and localized fibrous tumors. Am J Surg Pathol 1995;19:1124-1137.

12 Xiao SY, Rizzo P, Carbone M. Benign papillary mesothelioma of the tunica vaginalis testis. Arch Pathol Lab Med 2000;124:143-147.

13 Baker PM, Clement PB, Young RH. Malignant peritoneal mesothelioma in women: a study of 75 cases with emphasis on their morphologic spectrum and differential diagnosis. Am J Clin Pathol 2005;123:724-737.

14 Kerrigan SA, Turnnir RT, Clement PB, Young RH, Churg A. Diffuse malignant epithelial mesotheliomas of the peritoneum in women: a clinicopathologic study of 25 patients. Cancer 2002;94:378-385.

15 Barbera V, Rubino M. Papillary mesothelioma of the tunica vaginalis. Cancer 1957;10:183-189.

16 Chetty R. Well differentiated (benign) papillary mesothelioma of the tunica vaginalis. J Clin Pathol 1992;45:1029-1030.

17 Fujii Y, Masuda M, Hirokawa M, Matsushita K, Asakura S. A case of benign mesothelioma of the tunica vaginalis testis. Hinyokika Kiyo 1993;39:89-92.

18 Fukunaga M. Well-differentiated papillary mesothelioma of the tunica vaginalis: a case report with aspirate cytologic, immunohistochemical, and ultrastructural studies. Pathol Res Pract 2009;206:105-109.

19 Grove A, Jensen ML, Donna A. Mesotheliomas of the tunica vaginalis testis and hernial sacs. Virchows Arch A Pathol Anat Histopathol 1989;415:283-292.

20 Jones MA, Young RH, Scully RE. Malignant mesothelioma of the tunica vaginalis. A clinicopathologic analysis of 11 cases with review of the literature. Am J Surg Pathol 1995;19:815-825.

21 Mikuz G, Hopfel-Kreiner I. Papillary mesothelioma of the tunica vaginalis propria testis. Case report and ultrastructural study. Virchows Arch A Pathol Anat Histol 1982;396:231-238.

22 Attanoos RL, Griffin A, Gibbs AR. The use of immunohistochemistry in distinguishing reactive from neoplastic mesothelium. A novel use for desmin and comparative evaluation with epithelial membrane antigen, p53, platelet-derived growth factor-receptor, P-glycoprotein and bcl-2. Histopathology 2003;43: 231-238.

23 Cury PM, Butcher DN, Corrin B, Nicholson AG. The use of histological and immunohistochemical markers to distinguish pleural malignant mesothelioma and in situ mesothelioma from reactive mesothelial hyperplasia and reactive pleural fibrosis. J Pathol 1999;189: 251-257.

24 King J, Thatcher N, Pickering C, Hasleton P. Sensitivity and specificity of immunohistochemical antibodies used to distinguish between benign and malignant pleural disease: a systematic review of published reports. Histopathology 2006;49:561-568.

25 Mayall FG, Goddard H, Gibbs AR. P53 immunostaining in the distinction between benign and malignant mesothelial proliferations using formalin-fixed paraffin sections. J Pathol 1992;168:377-381.

26 Ramael M, Lemmens G, Eerdekens C, Buysse C, Deblier I, Jacobs W, van Marck E. Immunoreactivity for p53 protein in malignant mesothelioma and nonneoplastic mesothelium. J Pathol 1992;168:371-375.

27 Schonherr A, Bayer M, Bocking A. Diagnostic and prognostic value of Ki67 proliferation fraction in serous effusions. Cell Oncol 2004;26:57-62.

28 Sington JD, Morris LS, Nicholson AG, Coleman N. Assessment of cell cycle state may facilitate the histopathological diagnosis of malignant mesothelioma. Histopathology 2003;42:498-502.

29 Taheri ZM, Mehrafza M, Mohammadi F, Khoddami M, Bahadori M, Masjedi MR. The diagnostic value of ki-67 and repp86 in distinguishing between benign and malignant mesothelial proliferations. Arch Pathol Lab Med 2008;132:694-697.

30 Kato Y, Tsuta K, Seki K, Maeshima AM, Watanabe S, Suzuki K, Asamura H, Tsuchiya R, Matsuno Y. Immunohistochemical detection of GLUT-1 can discriminate between reactive mesothelium and malignant mesothelioma. Mod Pathol 2007;20:215-220.

31 Shen J, Pinkus GS, Deshpande V, Cibas ES. Usefulness of EMA, GLUT-1, and XIAP for the cytologic diagnosis of malignant mesothelioma in body cavity fluids. Am J Clin Pathol 2009;131:516-523.

32 Chen JL, Hsu YH. Malignant mesothelioma of the tunica vaginalis testis: a case report and literature review. Kaohsiung J Med Sci 2009;25:77-81.

33 Churg A, Cagle PT, Roggli VL. Diffuse malignant tumors of the serosal membranes. In: Silverberg SG, Sobin LH (eds). AFIP Atlas of Tumor Pathology: Tumors of the Serosal Membranes, Fourth Series, Fascicle 3 ARP PRESS: Silver Spring, Maryland, 2006, pp 33-72.

34 Spiess PE, Tuziak T, Kassouf W, Grossman HB, Czerniak B. Malignant mesothelioma of the tunica vaginalis. Urology 2005;66:397-401. 